- - - -مجلة علوم الرافين، المجلد 28، العدد 2عدد خاص بالمؤتمر العلمي الثالث لعلوم الكيمياء، ص1 -7، 2019 - - - -

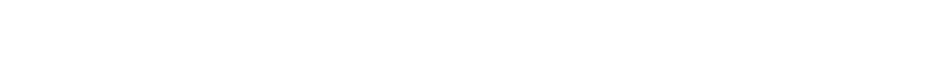

\author{
نكرى علي علوش \\ قصم الكيمياء/كلية العلوم/جلمعة الموصل علون \\ نول ننهن يونس \\ قمم العلوم التمريضية الأسلسية/كلية التمريض/جلمعة الموصل \\ رنافالفل جلم \\ قم الكيمياء/كلية التربية للبنت/جلمعة الموصل جلمل

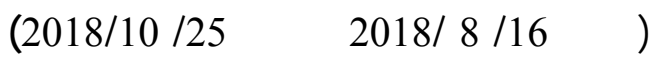 \\ المالخص
}

تضمن البهث درلسةسريريه لإنزيم الرينيز لدى مرضف العجز الكلوي وعلاقته ببعض المتغيرات الكيموحيوية ف مي

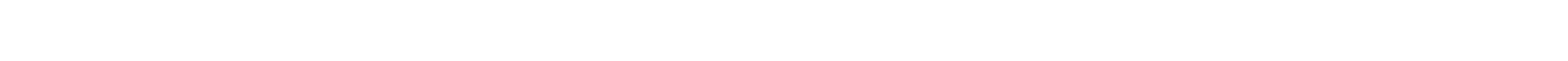

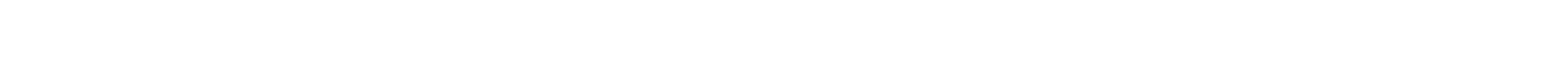

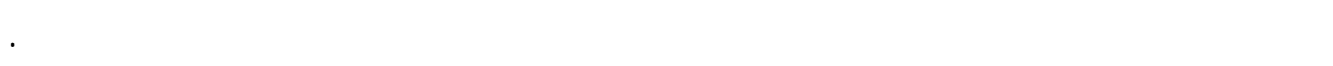
كما بينت النتائج وجود انغفاض معنوي في فعالية إذزيم الردينيز لدى الذكور مقارنة مع الانلث في لكل من مجم -وعتي المرضف والاصحاء. ودلست العلاقة ما بين فعالية إذزيم الردينيز والمتغيرت الكيموحيوية المقلسة بإيجاد معلفل الارتبطا الغطي. ووجدت علاقة خطيةسالبة بين فعالية إززيم الردينيز وفعالية انزيميسافيت اوكسديز وثايوسلفيت اوكسديز، وتركيز الألبومين وك ذالك

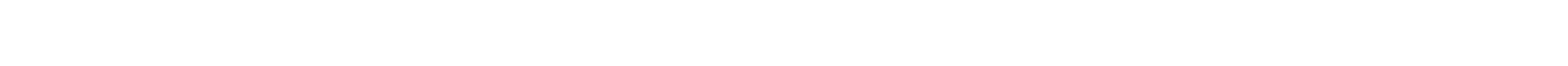
الكلوي.

يستنتج من البحث ان إذزم الردينيز يعد مؤثرا لمرض العجز الكلوي وله علاقة مع عدد من المتغيرات الكيموحيوي ـة لدى مجموعة المرضى.

الكاملت الدالة: الرينيز،سلفيت اوكسديز، ثايوسلفيت اوكسيز، الاريل لستريز، العجز الكلوي.

\title{
Clinical Study of Rhodanese Enzyme in Renal Failure Patients
}

Thikra A. Al-Allwsh

Department of Chemistry/ College of Science/ University of Mosul

Nawal Th. Younis

Department of Basic Nursing Science/ College of Nursing/University of Mosul

Rana F. Jasem

Department of Chemistry/ College of Education for Girls/ University of Mosul

\section{ABSTRACT}




$$
\text { نكرى علي علوش ولخرون }
$$

The research involved clinical study of rhodanese enzyme and its relation with some biochemical parameters in renal failure.

The result demonstrated a significant increase in the activity of rhodanese and metalloendopeptidase and in concentration of urea, and potassium while a significant decrease activity of sulfate oxidase, thiosulfate oxidase and arylesterase and in concentration of albumin and sodium in renal failure patients compared with control.

The results also demonstrated a significant decrease in the activity of rhodanese in male compared with female in each of patients and control groups.

Correlation coefficients between rhodanese and biochemical parameters of renal failure patients showed that activity of rhodanese has negative correlation with activity of sulfate oxidase, thiosulfate oxidase and concentration of albumin while appositive correlation with activity of metallo -endopeptidase and concentration of urea in renal failure patients.

These results provide evidence of a major role for rhodanese enzyme in renal failure, which led to consider it as a marker for renal failure and its relation with biochemical parameters in patients group.

Keywords: rhodanese, sulfate oxidase, thiosulfate oxidase, arylesterase, renal failure.

\section{اللقدمة}

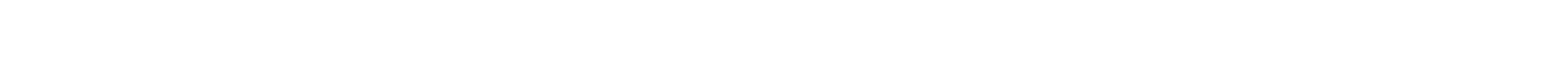

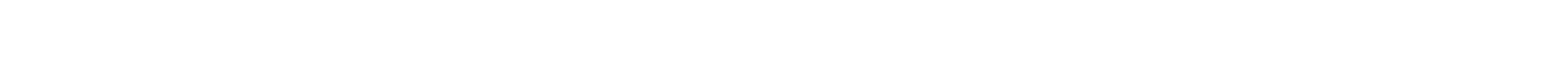

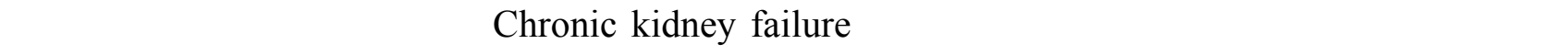
في تركيز بعض المتغيرات اللسريرية الموجوة في الدم (Mahendra et al., 2007).

يعد الردينيز Rhodanese إلى الثايوسيانيد Thiocayate الأفل ضررا مستخما Thiosulfate كماة أسلس وكما موضح ف في المعادل فة أدن له: Rhodanese (Allwsh and Mohammad, 2009)

$$
\mathrm{CN}^{-}+\mathrm{S}_{2} \mathrm{O}_{3}^{-} \longrightarrow \mathrm{SCN}^{-}+\mathrm{SO}_{3}
$$

ولهذا يلق عليه أيضا (Thiosulfate sulfur ransferase)، يؤدي إذزيم الرنييز دورا في تكوين بروتينت الكبريت

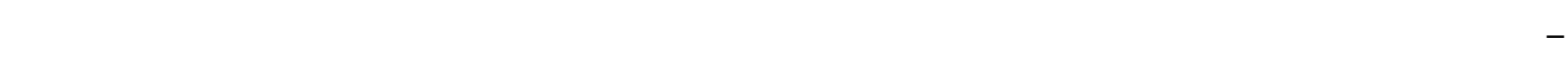
والمعدة (Cipollone et al., 2008).

وأثارت الدرلسك (Hasuike et al., 2004; Ansell and Lewis, 1970) إلى إن هذا الفاعل ض. -روري لإزالـ ـة سمية للسيانيد دلخل الجم وان تشيط إذزيم Rhodanese يؤدي إلى ترلمم ايونات الميانيد عبرسل سلة انتق لـ الالكترون ـات

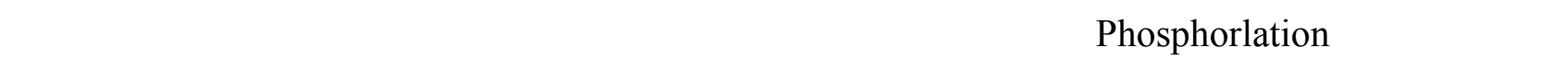
سمية للسيانيد وتحويله اله الثايوسيانيد الذي يطرح عنطريق الادرار واللعلب والعرق اله خارج الجيم.

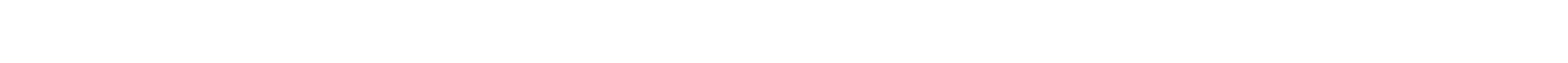
المتغيرك الكيموحيوية لدى المرضى.

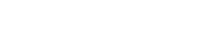

النماذج المستخمة:

قم جمع (40) نموذج م للأشخاص أصحاء تراومت أعمارهم بين (25-65 year) ولكلا الجنسن (22) نمونجا للنكور و

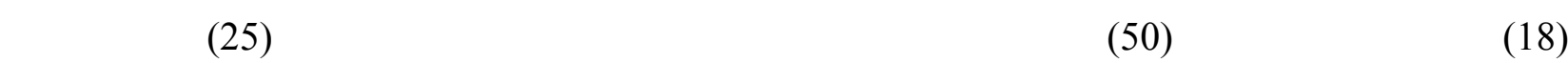


(25) تراومت أعمارهم بين (25 year-65) أيضا ولكلا الجنسن وقشخصت حالتهم من قل لطباء مختصن في مست شفى البنسينا التعليمي.

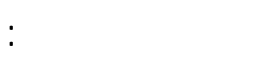
جمعت عينت الدم في أنابيب جلفة وظيفة في حملم مائي بدرجة 37 ولمدة 15 دقيقة بعدها قم فصل المحلول الرائق من الجزء المتخثر بجهاز الطرد المركزي بسرعة (4000g) ولمدة 10 دقائق حيث يمل المحلول الرائق مصل الهم (Burtis and Ashwood, 1999)

قفير المتغيرات الكيموحيوبة في مطل العم: مُ قيلس المتغيرات الكيموحيوية في مصل الم بلستخدلم الطرائق الموضحة في (الجدول 1) أننه.

الجطل 1: المتغيرات الكيموحيوبة المقلسة في مطل الم

\begin{tabular}{|c|c|c|c|}
\hline المصرر & طربقة الفيلس & المتغيرات الكيموحيوبة القلسة & ت \\
\hline $\begin{array}{c}\text { ( Urbanska et al., } \\
\text { 2002) }\end{array}$ & تكوين معقد الثايوسيانيد & فالية إذزيم Rhodanese U/ml & 1 \\
\hline (Woo et al., 2003) & بلستخدلم كلثف فيروسيانيد البوتلسيوم & sclfate oxidase U/ml فعالية & 2 \\
\hline ( Mark et al., 1972) & تكوين كلثف فيروسيانيد البوتلسيوم & فالية ف/miosulfate oxidase Ul & 3 \\
\hline (Tomas et al., 2000) & بلستخدلم مادة اللسبلس فنل لمسيتيت & $\begin{array}{c}\text { Arylesterase فالية } \\
\text { بmole /ml }\end{array}$ & 4 \\
\hline $\begin{array}{r}\text { (Kanazawa and } \\
\text { Johnston, 2007) }\end{array}$ & بلستخدلم مادة الإسلس الكازئين & فعetalloendopeptidase U/ml فعالية & 5 \\
\hline $\begin{array}{c}\text { (Annino and Giese, } \\
1976 \text { ) }\end{array}$ & بلستخدل كلثف البروموكريسول الخضر & Albumin Conce. $\mathrm{gm} / 100 \mathrm{ml}$ & 6 \\
\hline $\begin{array}{c}\text { (Toro and Ackermann, } \\
1975 \text { ) }\end{array}$ & بلستخدلم الطريقة الانزيمية & Urea Conce. $\mathrm{m} \mathrm{mol} / \mathrm{L}$ & 7 \\
\hline (Bishop et al., 2000) & بلستخدم قنيةطف الانبعاث باللهب & $\begin{array}{l}\text { Sodium, Potassium } \\
\text { Conce. } \mathrm{m} \mathrm{mol} / \mathrm{L}\end{array}$ & 8 \\
\hline
\end{tabular}

التحلل الإحصائ

قم إيجاد المعدل والانحرف القيلسي وتحليل النتائج إحصائياً بلستخدلم لختبار T-test لبيلن الاختلاف ب. ين مجم -وعتين وكذك مة إيجاد معالم الارتبط الخطي Correlation coefficients لبيان العلاقة بين فعالية إذ -زيم الرديني -ز والمتغي -رات الكيموحيوية المقلسة في مجموعة المرضى (Kirkwood, 1988).

\section{النتائج والمنلشة}

فعالية النزم الرنينيزفيمجموعت السيارة والمرضف

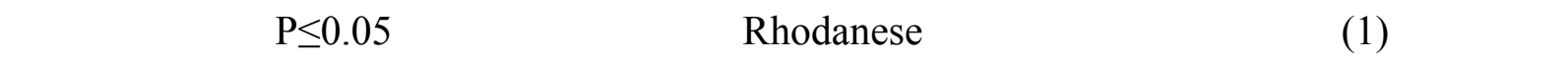
المرض مقارنة بمجموعة للسطرة وهذا ما لكه البلحثون (Hasuike et al., 2004 ; Allwsh and younis , 2010) زيادة نسبة الثايوسيانيد عندمرضف العجز الكلوي مما يشير إلى ارتفاع فعالية الازيم من تحويله للسيانيد اله الثايوسيانيد الأفل سمية، وان الخل في وظيفة الترشيح الكلوي لدى المرضف يؤدي إلى قلة لستخلاص الثايوسيانيد في الجسم. 


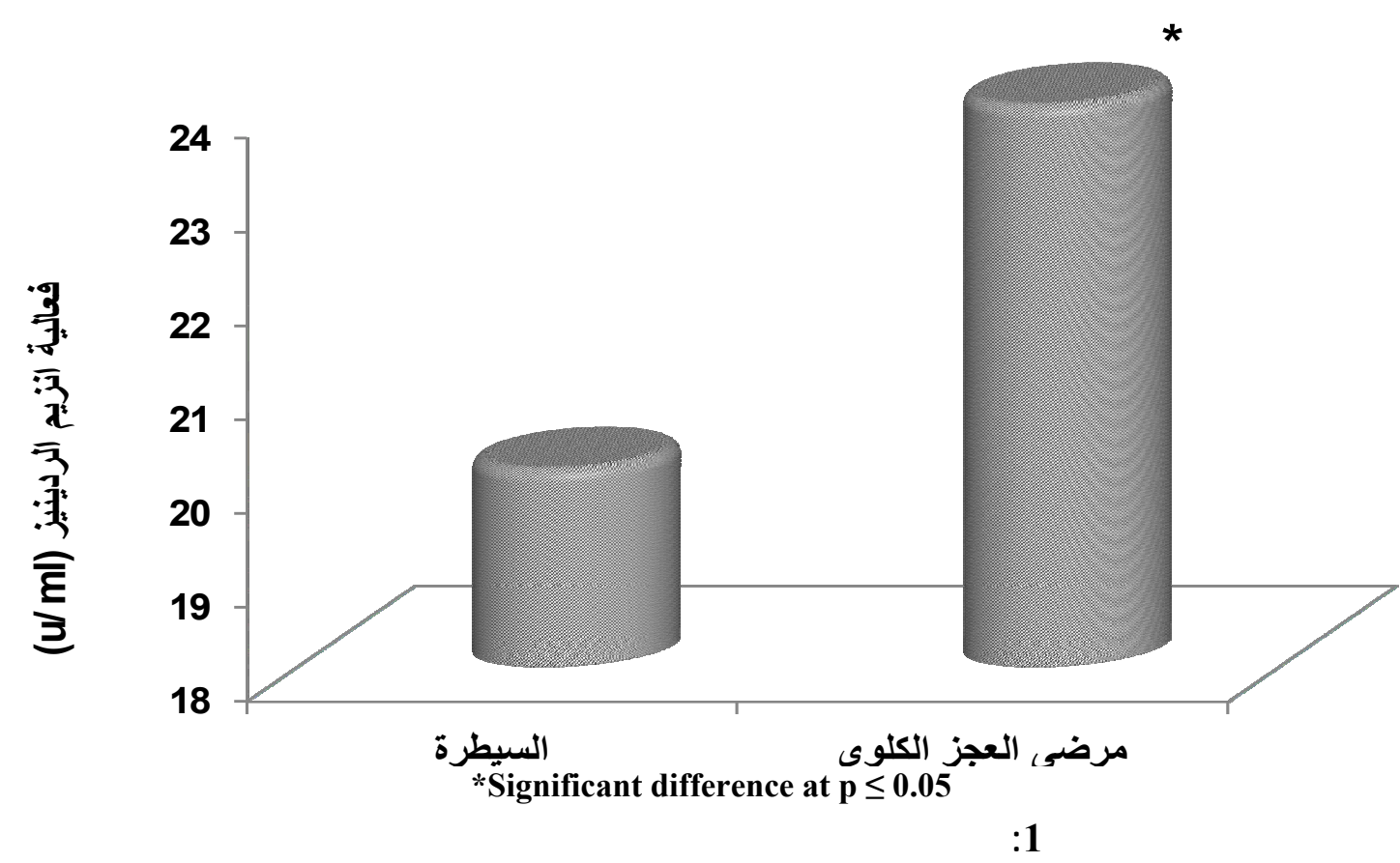

تألثير الجس علل فعالية إنزم الرنينيز:

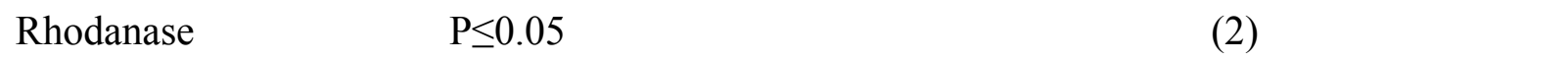

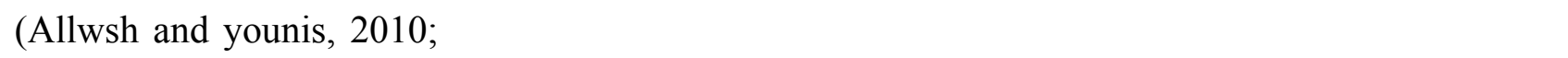

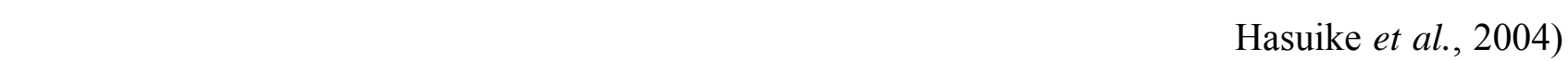

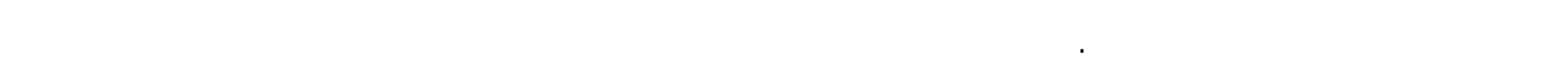
اللسيطرة.

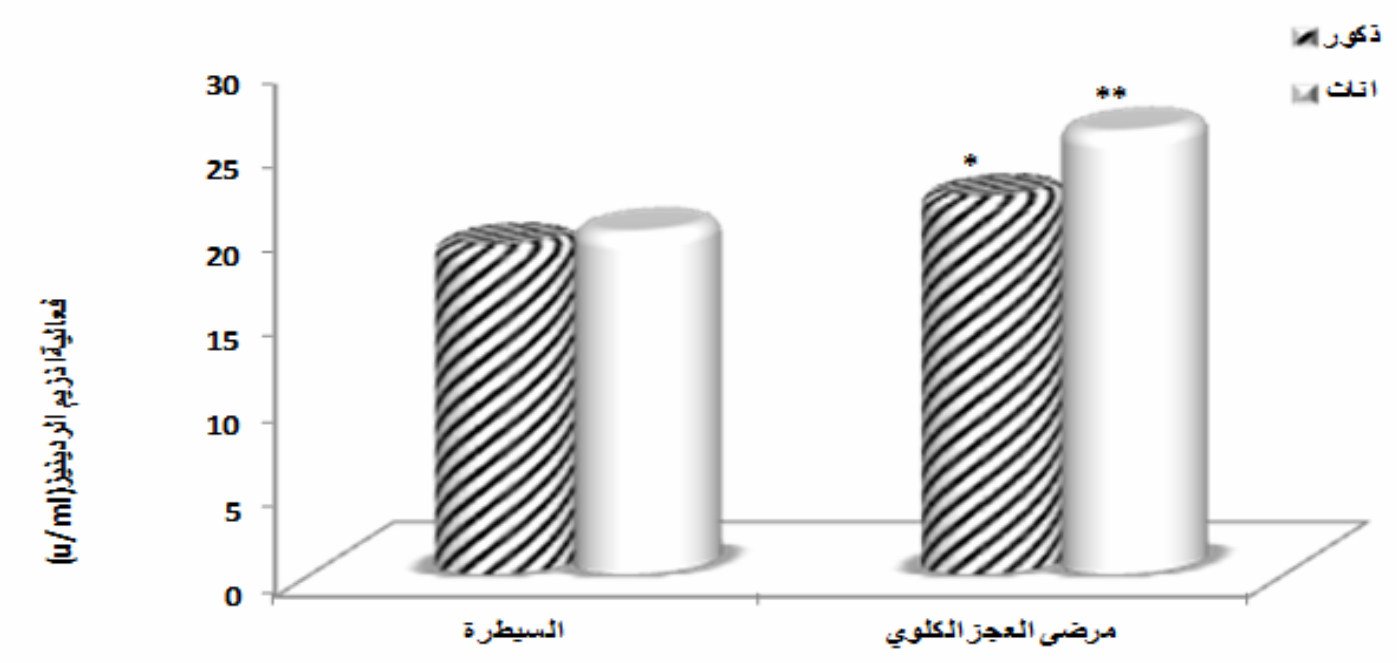

*Significant difference at $\mathbf{p} \leq 0.05, * *$ Significant difference at $p \leq 0.01$

الشكل 2: تأثير الجض علىفعالية إنزم الرنينيزفيمجموعت اللسمرة والمرضف 
المغيرل المربيرية المقلسة:

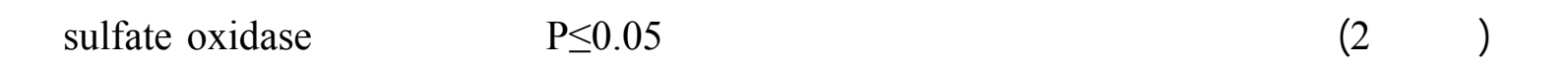

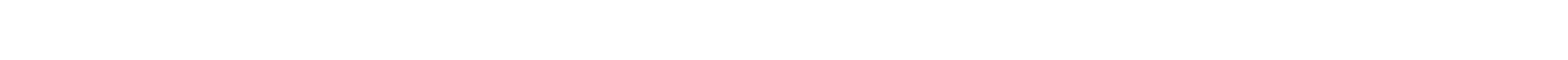

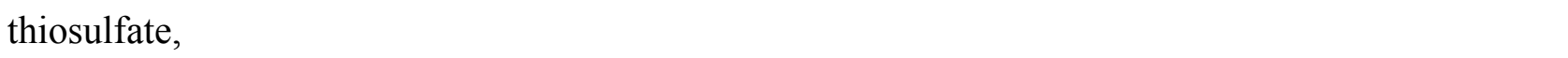
sulphocystein, sulfite

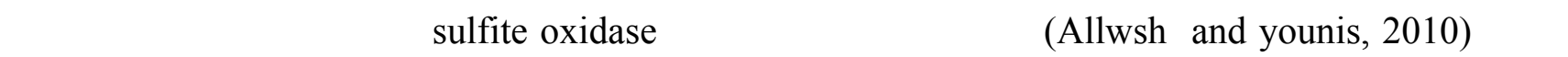
إلى زيادة نسبة الكبريتيت sulfite الذي يؤثر على النقل الالكترونت ضمن اللسلسلة التفسية وبالتالي يؤدي إلى انخفاض إنتاج

الطاقة علىشكل الينوسين ثلاثي الفوسفلت ATP في المايتوكوندريا ضمن الخلايا (Tan et al., 2005). كما يوضح (الجدول 2) أيضا وجود النخفاض معنوي في فعالية إذزيم thiosulfate oxidase عند مستوى الاحتمالية

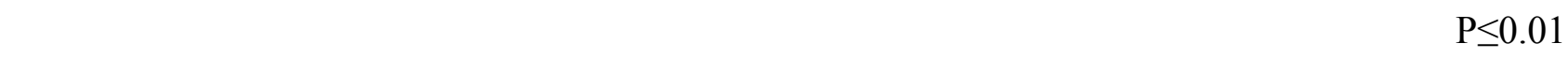
من زياة نسبة (Allwsh and younis, 2010) الإنزيم الذي يعل على لُهسة thiosulfate والنقليل منسميته.

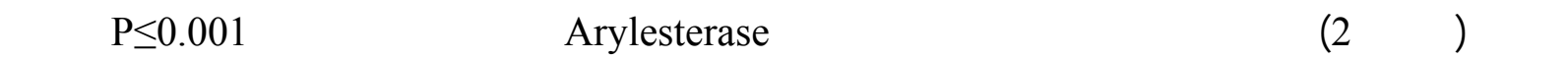

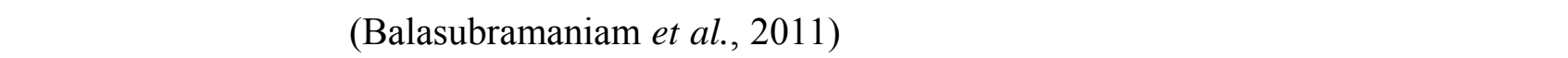
لدى مرضف العجز الكلوي.

\section{الجرل 2: المتغيرل الكيموحيوبة الفلسة في مطل مرمرض المجز الكلوي مقارنة مع مجموعة اللسيلرة}

\begin{tabular}{|c|c|c|c|}
\hline مجموعة مرضف المجز الكلوي & المعل مجموعة اللنحرل الفيلمي & المتغيرل الكيموحيوبة الفلسة & ت \\
\hline $0.16 \pm 0.28^{*}$ & $0.29 \pm 0.52$ & sulfate oxidase $\mathrm{U} / \mathrm{ml}$ & 1 \\
\hline $0.14 \pm 0.17 * *$ & $0.24 \pm 0.45$ & thiosulfate oxidase $\mathrm{U} / \mathrm{ml}$ & 2 \\
\hline $7.1 \pm 76.25 * * *$ & $5.67 \pm 116.5$ & Arylesterase $\mu$ mole $/ \mathrm{ml}$ & 3 \\
\hline $0.53 \pm 18.63 * *$ & $0.36 \pm 15.29$ & metalloendopeptidase U/ml & 4 \\
\hline $0.05 \pm 2.8 *$ & $0.03 \pm 4.11$ & Albumin gm/100ml & 5 \\
\hline $2.5 \pm 18.23 * * *$ & $0.8 \pm 5.61$ & Urea $\mathrm{m} \mathrm{mol} / \mathrm{L}$ & 6 \\
\hline $1.6 \pm 120 * *$ & $0.9 \pm 162.5$ & Sodium $\mathrm{m} \mathrm{mol} / \mathrm{L}$ & 7 \\
\hline $0.21 \pm 7.02 *$ & $0.09 \pm 5.2$ & Potassium $\mathrm{m} \mathrm{mol} / \mathrm{L}$ & 8 \\
\hline
\end{tabular}

*Significant difference at $\mathrm{p} \leq 0.05, * *$ Significant difference at $\mathrm{p} \leq 0.01$

$* * *$ Significant difference at $\mathrm{p} \leq 0.001$

والظهر (الجدول 2) أيضا ارتقاعا معنويا في فعالي مة إذ -زيم metalloendopeptidase عن ـد مـ ستوى الاحتمالي ـة

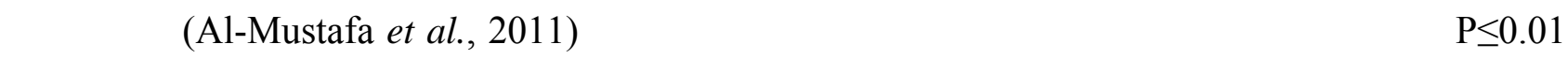

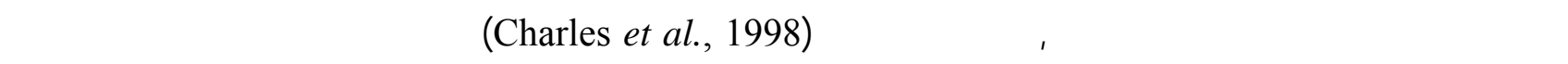

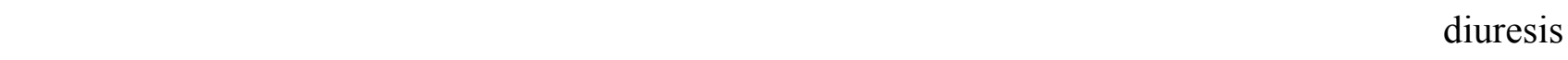
الالدوستيرون المضاد للإدرار.

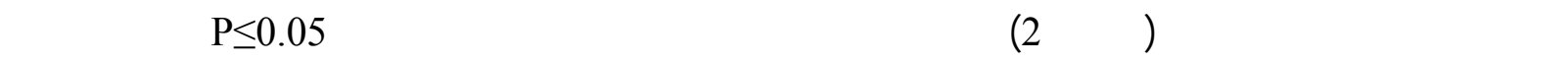
الألبومين لدى مجموعة المرض مقارنة بمجموعة للسطرة وربما يعود للسبب إلى قلة البروتين في الغذاء المتناول أو إلى قلة

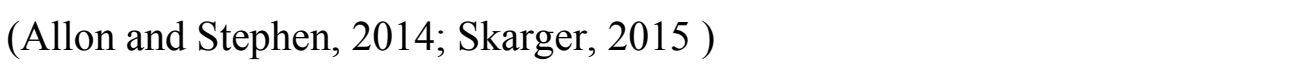


ويشير (الجدول 2) إن هنك ارتقاعا معنويا في تركيز اليوريا عند مستوى الاحتمالية P>0.001 في مصل م مجموعة

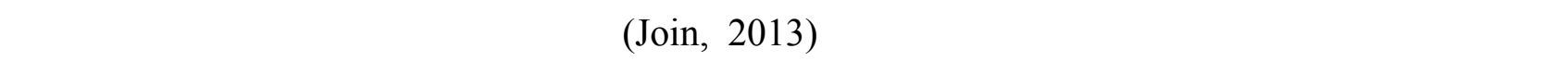
العجز الكلوي نتيجة القصور في تششبح الكلى.

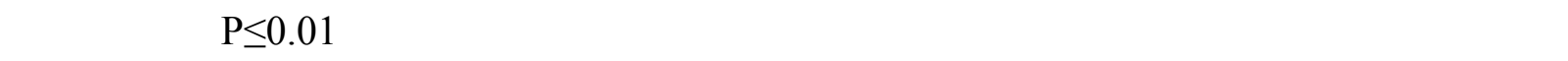

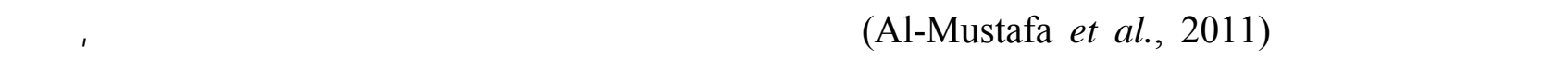

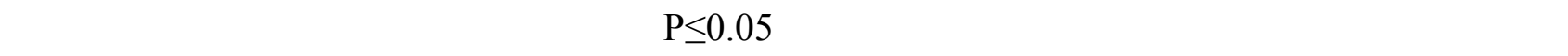
للسطرة وربما بسب العلاقة العكسية ما بين الايونين (Giorgina et al., 2010).

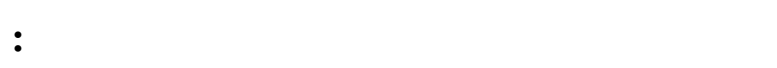

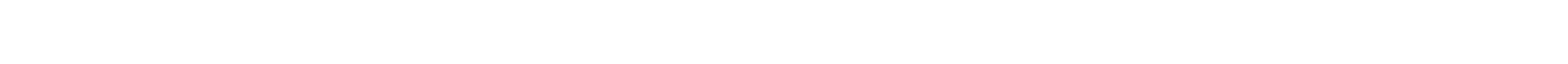

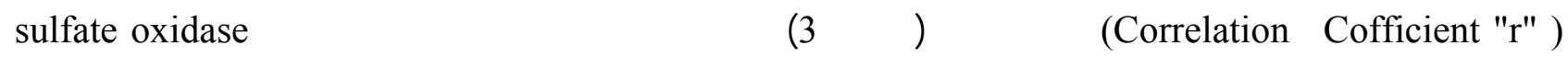

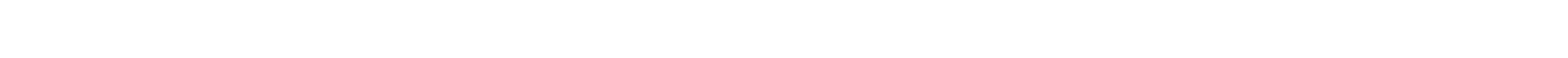

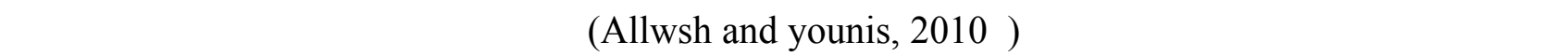
مع thiosulfate oxidase كما موضح في (الجدول 3) وذلك لانخفاض فعالية الإنزيم عندمجموعة مرضف العجز الكلوي مما

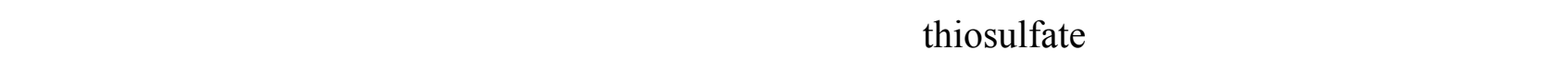

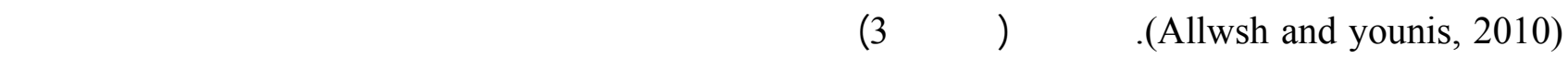

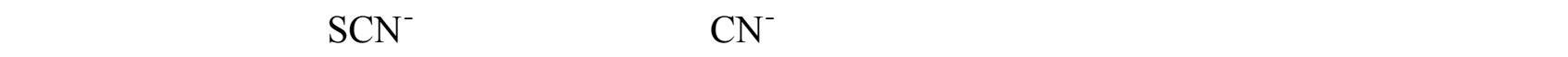

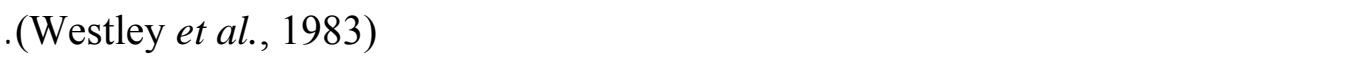

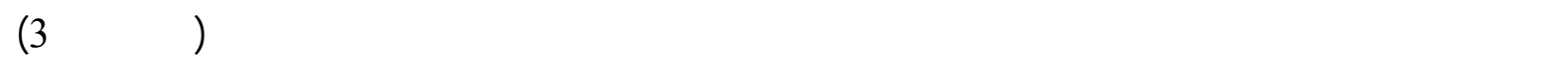

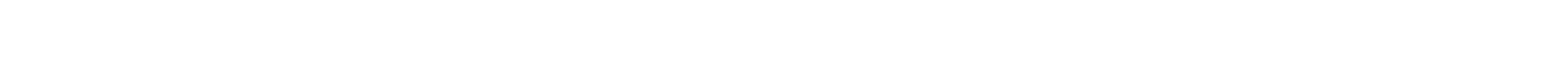

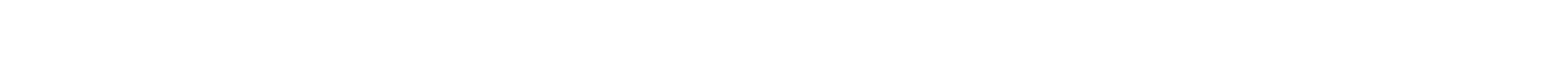
على ارتفاع فعالية إذزيم الردينيز للقليل منسميته (Allwsh and younis, 2010).

الجرط 3: الللفة بما بن إنزم الرينيز والمتغيرات السربرية المقلسة فمجموعت الليمرة والمرضف

\begin{tabular}{|c|c|c|c|c|c|c|c|c|c|}
\hline المتغيرات & Rhodanese & $\begin{array}{c}\text { Sulfate } \\
\text { Oxidase }\end{array}$ & $\begin{array}{c}\text { Thiosulfate } \\
\text { oxidase }\end{array}$ & $\begin{array}{c}\text { Metall } \\
\text { endo } \\
\text { peptidase }\end{array}$ & $\begin{array}{c}\text { Aryl } \\
\text { esterase }\end{array}$ & $\mathrm{Na}$ & $\mathbf{K}$ & Urea & Albumin \\
\hline مجموعة للسطررة & 1.000 & 0.132 & 0.034 & 0.073 & 0.041 & 0.155 & 0.137 & 0.157 & 0.172 \\
\hline مرضى الفثل & 1.000 & $\begin{array}{c}* \\
-0.0411\end{array}$ & $\begin{array}{c}* \\
-0.047\end{array}$ & $\begin{array}{c}* \\
0.0357\end{array}$ & 0.240 & 0.221 & 0.118 & $\begin{array}{c}* \\
0.463\end{array}$ & $\begin{array}{c}* \\
0.045-\end{array}$ \\
\hline
\end{tabular}

*Correlation is significant at $\mathrm{p} \leq 0.05$

$$
\text { المصد - الر }
$$

Allon, N.F.; Stephen, Z.F. (2014). Reassessment of albumin as a nutritionl marker in kidney. American Soc. Nephrol., 21(2), 223-230.

Annino, J.S.; Giese, R.W. (1976). "Clinical Chemistry". $4^{\text {th }}$ ed., Little, Brown and Co., Boston. 177, $183,186$.

Al-Mustafa, L.S.; Allwsh, Th.A.; AL-Jarah, I.A. (2011). Biochemical study of metallo endopeptidase and its relation with chronic renal failure. Raf. J. Sci., 22(4), 71-87.

Allwsh, Th.A.; Mohammad, Z.H. (2009). Biochemical study of rhodanese enzyme isolated from blood and its role in cyanide detoxification. Raf. J. Sci., 20(1), 61-80. 
Allwsh, Th.A.; Younis, N.Th. (2010). Biochemical study of thiocyanate and its relation with renal failure patients. Tikrit J. Pure Sci., 15(3), 241 -246.

Ansell, M.; Lewis, F.A.S. (1970). A review of cyanide concentration found in human organs. A surrey of literature concerning cyanide metabolism "normal", non- fetal, and fetal body cyanide levels. J. Forensic Medicine., 17, 148-155.

Balasubramaniam, G.; Anand, U.; Duraisamy, V. (2011). Serum paraoxonase (Arylesterase) activity in chronic renal failure. J. Medicine., 12(2011), 17-20.

Bishop, M.L.; Duben-Engelkirk, J.; Fody, E.P. (2000). "Clinical Chemistry: Principle, Procedures, Correlation". $4^{\text {th }}$ ed., Lippincott Williams and Wilkins, Philadelphia.

Burtis, C.A.; Ashwood, E.R. (1999). " Tietz Text Book of Clinical Chemistry". $3^{\text {rd }}$ ed., W.B. Saunders company, USA, 1037.

Charles, J.F.; James, C.S.; William, G.H.; Dauid, J.W. (1998). Inhibition of neutral endopeptidase causes vasoconstriction of human Resistance Vessels in vivo. American Heart Association. 97, 2323-2330.

Cipollone, R.; Ascenzi, P.; Tomao, P.; Imperi, F.; Visca, P. (2008). Enzymatic detoxification of cyanide clues from pseudomonas aeruginosa Rhodanese. J. Mol. Microbiol. Biotechnol., 15(2-3), 199-211.

Giorgina, B.P.; Marco, C.; Lorenzo, O.; Maria, C.D.; Valentina, C.; Giulio, R. (2010). Acute renal failure, sever sodium and potassium imbalance and sudden tetraplegia. Clin. Kidney J. 3, 247252.

Hasuike, Y.; Nakanishi, Y.; Moriguchi, R.; Otaki, Y.; Nanami, M.; Hama, Y.; Naka, M.; Miyagawa, K.; Izumi, M.; Takamitsu, Y. (2004). Accumulation of cyanide and thiocyanate in haemodialysis patients. Nephrol. Dial. Transplant., 19(6), 1474-1479.

Join, U.S. (2013). Symptoms of high blood urea nitrogen in kidney failure. Search Kidney Failure., 17, 42.

Kanazawa, M.; Johnston, C. (2007). Distribution and inhibition neutral metallo endopeptidase (NEP) (Ec 3.4.24.11), the major derivative enzyme for atrialnatriuretic pepide, in the rate kidney. Clin . Exp. Pharm. Physiol., 18(6), 449-453.

Kirkwood, B.R. (1988). "Essentials of Medical Statistics". $1^{\text {st }}$ ed., Black well Scientific Publication, Oxford; pp. 43-56.

Mahendra, A.; Rajiv, G.; laural, M. (2007). Acute renal failure. Nephrology., 20, 1-11.

Mark, R.; Hall, R.; Berk, S. (1972). Thiosulfate oxidase from an Alcaligenes grown on mercaptosuccinate. Canadian J. Microbiol., 18(2), 235 -245, 10.1139/m 72- 036.

Skarger, A.G. (2015). Hypoalbuminemia in renal failure: pathogenesis and therapeutic considerations. Kidney Blood Press Res., 28, 307-310.

Tan, W.H.; Eichler, F.S.; Hoda, S.; Lee, M.S.; Baris, H.; Hanley, C.A.; Shih, V.E. (2005). Isolated sulfite oxidase deficiency :a case report with a novel mutation and review of the literature. Pediatrics., 116(3), 757-766.

Tomas, M.; Senti, M.; Gareia- Faria, F.; Vila, J.; Torrents, A.; Coves, M.; Marragot, J. (2000). Effect of simrastatin therapy on paraxonase activity and related lipoprotein in familial hypercholesterolemic patients. Arterioscler. Thromb Vasc. Biol., 20, 2113.

Toro, G.; Ackermann, P.G. (1975). "Practical clinical chemistry." $1^{\text {st }}$ ed., printed in the United state of America, pp. 271-273, 143-145.

Urbanska, A.; Lezczynski, B.; Matok, H.; Dixon, A. (2002). Cyanide detoxification enzymes of bird cherryoat aphid. Electronic. J., 5(2), 334-337.

Westley, J.; Adler, H.; Westley, L.; Nishida (1983). The sulfurtransferase. Fundamental and Applied Toxicol., 3, 377-382.

Woo, W.H.; Yang, H.; Wong, K.P.; Halliwell, B. (2003). Sulphite oxidase gene expression in human brain and in other human and rat tissues. Biochem. Biophys. Res. Commun., 305, 619 -623. 